\title{
STRUCTURE OF COMETS AND THE POSSIBLE ORIGIN OF FAINT ASTEROIDS
}

\author{
V. VANÝSEK \\ Charles University \\ Prague
}

\begin{abstract}
It is shown that very old and still active icy-conglomerate or "clathrate" cometary nuclei may exist at the outer boundary of the asteroidal belt and belong to the group of relatively stable "outer" short-period comets of which only a small fraction have been discovered or recognized as cometary objects.

The search for these comets among very faint asteroids with the mean motion $\mu \leqslant 600^{\prime \prime}$, and the study of their behavior could be very important to our knowledge about the final stage of cometary nuclei.
\end{abstract}

The study of the processes involved in the formation of the solar system requires some attention to the physical structure of comets, as has been emphasized by many authors in the last two decades. It appears to be a logical procedure to follow the formation of comets as a link to a better understanding of the processes that occurred in the early stages of the solar system because it is highly probable that comets represent the remnants in which preplanetary matter is preserved.

The comets are "soft" bodies and, unlike other sizable objects in the solar system, undergo relatively very fast changes. The outflow of gases and dust from cometary nuclei, bright outbursts, and splitting of comets are indisputable evidences of processes that lead to complete disintegration or to considerable changes of the structure of the nucleus in the time range, which depends on the perihelion distance, original mass, and orbital period.

The mean lifetime of a cometary nucleus need not be necessarily considered as the lifetime of its entire existence but as the time range in which such a body possesses the ability to produce observable typical cometary phenomena, which distinguish the cometary appearance from the asteroidal one. Besides complete disintegration, the asteroidal appearance might be another possible final stage of a cometary nucleus. The existence of asteroids like Icarus, Geographos, Apollo, Adonis, and Hermes or the Hilda group supports this idea, which has been discussed by many authors in the past.

It is evident, however, that the final stage of a cometary nucleus is determined by its composition and structure, as well as by the formation 
process. What is the final stage (is it the starlike appearance of exhausted remnants of the nuclei or is it complete disintegration) is one of the most significant questions concerning the evolution of comets.

The high abundance of neutral hydrogen in the cometary atmosphere, confirmed by observations of the resonance Lyman- $\alpha$ radiation and strong $\mathrm{O}-\mathrm{O}$ bands of $\mathrm{OH}$ of recent bright comets (Code and Houck, 1970), indicates that hydrogen compounds are dominant constituents of the cometary nuclei. The most probable precursor of atomic hydrogen and hydroxyl is $\mathrm{H}_{2} \mathrm{O}$.

Although there is still lack of direct evidence of the presence of water in comets, ${ }^{1}$ the $20 \mathrm{yr}$ old icy-conglomerate model proposed by Whipple (1950, 1951) and modified by Urey (1952), Donn and Urey (1957), and recently by Delsemme and Miller (1970) seems to be the best approach to the real composition of the cometary nuclei.

The homogeneous icy-conglomerate nucleus undergoes complete disintegration. On the other hand, the "dead" nucleus requires a more complicated initial structure of comets. The possibility of some kind of stratification in the nucleus is supported by the fact that the "new" comets (according to Oort's terminology) or those with low frequency of perihelion passages produce large amounts of nonvolatile material and gases, a fact that is not typical of short-period comets. This may imply that the young nucleus is surrounded by a loose layer of dust and ice grains. This loose layer requires a lower amount of energy for evaporation than deeper parts of nuclei and it easily produces a very extensive dust-gaseous coma.

One can assume either the nonvolatile material surrounded by the mantle of volatile material or the increasing compactness of a matrixlike structure of the nonvolatile material toward the center of the nucleus.

As was shown by Whipple and Stefanik (1965), the radioactive heating of an icy-conglomerate nucleus can transfer the volatile material such as $\mathrm{CH}_{4}$ from the center to the surface layers. The condensed $\mathrm{CH}_{4}$ can form a brittle shell easily breakable by the heat shock shortly before the first approach to the perihelion. This breakage can be observed as a splitting of the nucleus and seems to be very typical for the "new" comets (Harwit, 1968; Pittich, 1971; Stefanik, 1965).

On the other hand, we have little knowledge about the behavior of the "dying" comets. ${ }^{2}$ The often-studied secular changes of comet $P /$ Encke must be interpreted with precaution because this object is not quite typical among short-period comets.

Comets in the transient stage, with nearly depleted nuclei, are probably more numerous just at the mean heliocentric distances beyond which a comet nucleus can survive without considerable diminution of the original mass.

\footnotetext{
${ }^{1}$ See, however, p. 461.

${ }^{2}$ See p. 427.
} 
If $t_{0}$ is the mean lifetime for a nucleus in the circular orbit at the heliocentric distance $r=1 \mathrm{AU}$, then the lifetime $t=t(r)$ at $r>1$ is given approximately by the relation

$$
\frac{t}{t_{0}}=\frac{z_{0}}{z_{r}}
$$

where $z_{0}$ and $z_{r}$ are vaporization rates at corresponding heliocentric distances, defined by

$$
z_{r}=p\left(2 \pi m T_{r}\right)^{-1 / 2}
$$

where $p=p(T)$, the saturated vapor pressure; $m$ is the mass; and $T_{r}$ is temperature which holds for the energy balance equation

$$
F_{0} r^{-2} \alpha=\sigma T_{r}^{4} \epsilon+z_{r} L
$$

where $F_{0}$ is solar energy flux at $r=1, \alpha$ and $\epsilon$ are coefficients of absorptivity and emissivity of the nucleus, and $L=L(T)$ is the latent heat of vaporization. The vaporization rates for various homogeneous nuclei composed from various constituents were computed by Huebner (1965).

The $t$ for a comet in the elongated elliptical orbit must be computed as a cumulative effect of the vaporization and restitution of the volatile material in surface layers before and after perihelion passages. However, for a comet in a less eccentric orbit, formula (1) can be used with the average value of $r$.

Figure 1 shows the relative lifetime $t / t_{0}$ of a nucleus composed of $\mathrm{H}_{2} \mathrm{O}$, $\mathrm{CO}_{2}$, and $\mathrm{CH}_{4}$ as a function of the heliocentric distance. The nucleus is

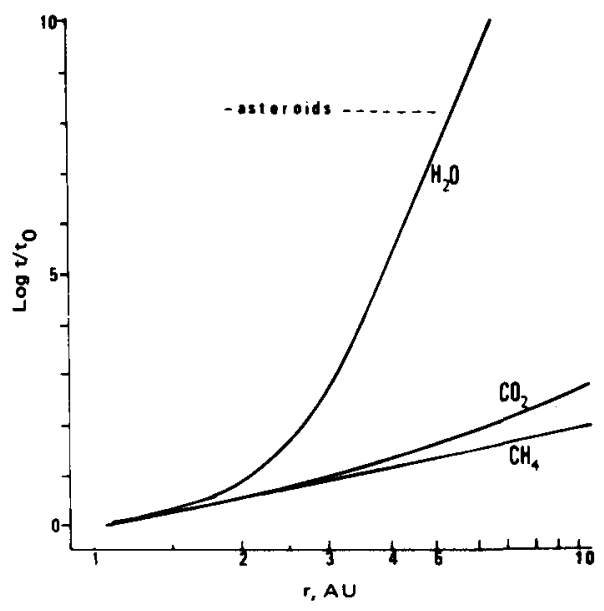

ligure 1.- The relative lifetime $t / t_{0}$ for ices of some simple compounds as a function of the heliocentric distancc. The extension of the asteroidal belt is marked. 
supposed to be a spherical rotating body with the albedo 0.1 in a circular orbit. (The effect of possible collisions in the asteroidal belt and the influence of the corpuscular solar radiation are not considered, therefore the value of $\log t / t_{0}$ in $r=2.5$ to $3.5 \mathrm{AU}$ might be somewhat overestimated.)

The curve for the $\mathrm{H}_{2} \mathrm{O}$ ice model can be used with good approximation for an icy lattice of the clathrates, as far as the hydrocarbons and radicals are only a minor contamination in the ordinary ice. If the mean production rate of a periodic comet is about $10^{29}$ to $10^{30}$ molecules $\mathrm{s}^{-1}$, the real mean value of $t_{0}$ ranges between $10^{3}$ and $10^{4} \mathrm{yr}$, depending on the initial mass, frequency of perihelion passages, and the lifetime for an icy-conglomerate nucleus at $r \simeq 3.5$, which is on the order of $10^{8}$ to $10^{9} \mathrm{yr}$. Even if this value seems to be somewhat unrealistically high, there is no doubt that the nucleus of a typical comet will be preserved for a very long time if it orbits beyond the asteroidal belt in the period that does not make simple commensurability ratios to Jupiter's mean motion.

Kresák $(1965,1971)$ pointed out that commensurabilities in the system of short-period comets with typical eccentricities frequently mean drastic changes in orbits because of the repetition of the close approach to Jupiter (which is not the case in asteroidal systems). Particularly the $1: 2$ ratio commensurability is "forbidden" as can be demonstrated by the outstanding gaps near the mean motion $\mu=600^{\prime \prime}$. According to Kresák, the commensurability gap $1: 2$ separates a compact "outer" group of comets from "inner" comets with a higher eccentricity of orbits and short perihelion distances. The "inner" comets have a short life and represent only a small fraction of the existing number of objects classified generally as short-period comets.

Therefore, if the icy-conglomerate or "clathrate" models are correct, a large number of the active comet nuclei with the mean motion $\mu<590^{\prime \prime}$ remain undiscovered until now, even if they are potentially observable as asteroidlike objects.

As one such example, the case of comet P/Oterma might be noted; it was a starlike object of the 17 th to 19 th magnitude before the last change of the orbit with orbital elements resembling those of an asteroidal orbit (e.g., 153 Hilda). Because of a change in its orbit in 1962, the comet became very faint and practically unobservable. Bouška (1965), using the $r^{-6.8}$ law for the brightness, obtained apparent magnitudes 22 to 27 mag; i.e., beyond detectability. However, when the gas production fades out, the $r^{-2}$ law is more realistic and the nucleus with low activity of an Oterma-type comet might be still detectable with large Schmidt telescopes. Moreover, the brightness outbursts, very typical for the giant comet Schwassmann-Wachmann 1, could be assumed as general behavior of comets at larger heliocentric distances. These outbursts (or surges) mean a considerable increase in brightness, and the comet becomes an easily observable object for a long time interval. As was shown recently by Pittich (1969), there exist at least 40 cases where comets appear to have suddenly increased their brightness shortly before their discovery. The 
increase of brightness occurs even at large heliocentric distances. In addition to comet Schwassmann-Wachmann 1,10 other cases with at least one outburst are known.

The frequency of the outbursts increases with the decrease of brightness amplitudes, and the nonperiodic changes in magnitudes of very faint starlike comets with asteroidal appearance might be expected as very typical.

The search for old but still partly active cometary nuclei among very faint asteroids would appear to be a worthwhile program, especially among the asteroids of the Palomar-Leiden survey. Because of the differences in cometary and asteroidal orbits, one can assume that some families and streams (in the sense of Alfvén's streams) of long-life comets with asteroidal appearance exist and can be distinguished from asteroids not only by occasional brightness flares but also by the grouping of orbital elements.

\section{REFERENCES}

Bouška, J. 1965, The Collisions of Asteroids. Bull. Astron. Inst. Czech. 16, 358.

Code, A. D., and Houck, T. E.1970, OAO Observations of Comet 1969g and Comet $1969 \mathrm{i}$. Bull. AAS 2, 321.

Delsemme, A. H., and Miller, D. C. 1970, Physico-Chemical Phenomena in Comets. II. Gas Adsorption in the Snows of the Nucleus. Planet. Space Sci. 18, 717-730.

Donn, B., and Urey, H. C. 1957, Chemical Heating Processes in Astronomical Objects. Mém. Soc. Roy. Sci. Liège Ser. 4, vol. 18, pp. 124-132.

Har wit, M. 1968, "Spontaneously" Split Comets. Astrophys. J. 151, 789-790.

Huebner, W. F. 1965, Über die Gasproduktion der Kometen. Z. Astrophys. 63, 22.34.

Kresák, L. 1965, On Two Aspects of Evolution of Short-Period Comets. Mém. Soc. Roy. Sci. Liège Ser. 5, vol. 12, pp. 459-467.

Kresák, L. 1971, The Dividing Line Between Cometary and "Asteroidal Orbits." The Motion, Evolution of Orbits and Origin of Comets, IAU Symp. 45. D. Reidel. Dordrecht. In press.

Pittich, E. M. 1969, Sudden Changes in the Brightness of Comets Before Their Discovery. Bull. Astron. Inst. Czech. 20, 251-292.

Pittich, E. M. 1971, The Motion, Evolution of Orbits and Origin of Comets, IAU Symp. 45. D. Reidel. Dordrecht. In press.

Stefanik, R. P. 1965, On Thirteen Split Comets. Mém. Soc. Roy. Sci. Lì̀ge Ser. 5, vol. 12, pp. 29-32.

Urey, H. C. 1952, The Planets. Yale Univ. Press. New Haven.

Whipple, F. L. 1950, A Comet Model. I. The Acceleration of Comet Encke. Astrophys. J. $111,375-394$.

Whipple, F. L. 1951, A Comet Model. II. Physical Relations for Comets and Meteors. Astrophys. J. 113, 464-474.

Whipple, F. L., and Stefanik, R. P. 1965, On the Physics and Splitting of Cometary Nuclei. Mém. Soc. Roy. Sci. Liège Ser. 5, vol. 12, pp. 33-52. 